\title{
Inelastic Response of an Overhanging Steel Beam
}

\author{
[ Antoine N. Gergess ]
}

\begin{abstract}
Mechanical loads are sometimes used to bend steel beams for curving. For this purpose, concentrated forces are applied to deform the beam about its weak axis. This paper derives equations to define the load versus residual deformation response for overhanging steel beams. Idealization of the circular curve based on the deformed shape is also presented. The process is verified by a numerical example.
\end{abstract}

Keywords —inelastic, steel, deformation, beam

\section{Introduction}

Cold bending is a process that is commonly used for curving steel beams about its weak axis [1]. The deformed shape of the beam is accomplished based on the residual deformations that sustain after removing the bending load. For this purpose, the steel beam is bent into the elasto-plastic range. Various bending procedures are available: (1) single point bending [2] and (2) two-point bending for simply supported beams [3]. In the elasto-plastic range, the residual deformation after removing the loads is determined by adding the inelastic deformation based on post-yield response of steel and subsequent elastic deformation from unloading.

This paper presents closed form equations for calculating residual inelastic deformation for an overhanging steel beam subject to a single concentrated load at the tip of the cantilevered portion. Simple mechanics principles are used in the derivations based on two successive integration of the curvature [4].

\section{Description}

\section{A. Set-up}

In the process, the steel beam is placed on simple supports as shown in Fig. 1. The length of the beam is $L$ and the overhanging portion is labelled as $a$. The mechanical load $P$ is applied as the tip of the cantilever to curve the beam. In practice, proprietary machines are developed by fabricators for this purpose [5].

The main objective of this paper is to determine the permanent deformed shape of the beam after removing the load $P$ and come up with a configuration that ensures close approximation to a circular shape. This will make it possible for steel fabricators to optimize the cold bending operation by selecting the most efficient loading configuration.

Antoine N. Gergess, PhD, PE, F.ASCE

University of Balamand

Lebanon

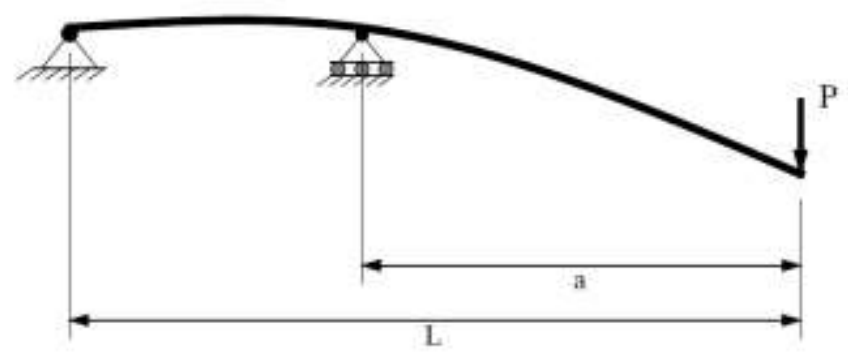

Figure 1. Beam layout

\section{B. Steel Beam Properties}

The steel beam consists of a symmetrical rolled $\mathrm{W}$ shape [6] with a flange width $h$ and thickness $b$. Since the steel beam is bent about its weak axis, the web effects are neglected. Consequently, each flange is analyzed separately as a rectangular beam of width $h$ and thickness $b$ as shown in Fig. 2.

Steel properties consist of its modulus of elasticity $E$ and its yield stress $\sigma_{y}$. The steel stress-strain $(\sigma-\varepsilon)$ curve is shown in Fig. 3. The elastic modulus of elasticity is defined as $E$ while in the elasto-plastic range it is defined as $E_{t}$.

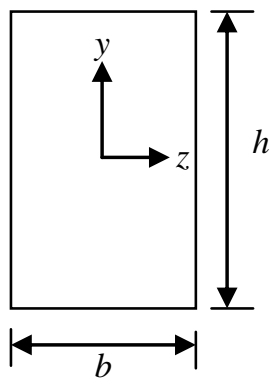

Figure 2. Steel beam flange dimensions

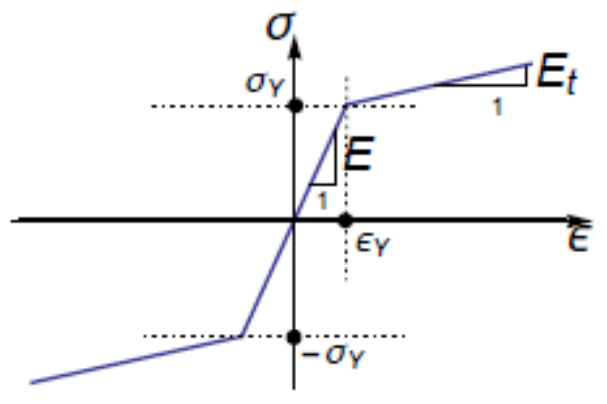

Figure 3. Stress-strain curve 


\section{Formulation}

To calculate deformations, strain-curvature relations need to be obtained based on simple mechanics principles. For this purpose, it is assumed that plain sections normal to the centerline remain plane and perpendicular lines to the centerline remain normal. This implies that the normal (bending) strain $\varepsilon$ relates to curvature $\kappa$ as given by (1):

$$
\varepsilon=\kappa \mathrm{y}
$$

where $y$ is the ordinate of the cross-section that is in the direction of the deformation as shown in Fig. 2.

\section{A. Stress-Strain Relationships}

Stress $(\sigma)$-strain $(\varepsilon)$ relationships are defined by $(2 \mathrm{a})$ and (2b) as follows:

$$
\begin{gathered}
\sigma=E \varepsilon \text { if } \sigma<\sigma_{y} \\
\sigma=\sigma_{y}+E_{t}\left(\varepsilon-\varepsilon_{y}\right) \text { if } \sigma \geq \sigma_{y}
\end{gathered}
$$

The physical description of the above relations is that the stress increases with strain proportional to Young's modulus $E$ up to the yield stress $\sigma_{y}$. After yield, the slope of the stressstrain curve is reduced to the hardening slope $E_{t}$ as shown in Fig. 3 [7].

Using (1), the stress-strain relationships ((2a) and (2b)) are cast as a function of the curvature (replace the strain $\varepsilon$ by $\kappa y$ ) which leads to (3a) and (3b) as follows:

$$
\begin{gathered}
\sigma=E \kappa y \text { if } \sigma<\sigma_{y} \\
\sigma=\sigma_{y}+E_{t}\left(\kappa y-\sigma_{y} / E\right) \text { if } \sigma \geq \sigma_{y}
\end{gathered}
$$

\section{B. Bending Moments}

The bending moment $M$ that develops from internal stresses is obtained by integrating the stress $\sigma$ multiplied by the moment arm $y$ from Fig. 2 as shown in (4) below:

$$
\begin{aligned}
M= & b \int_{-h / 2}^{h / 2} \sigma y d y=E_{t} I \kappa y+1 / 4\left(1-E_{t} / E\right) b h^{2} \sigma_{y} \\
& -1 / 3\left(1-E_{t} / E\right)\left(\sigma_{y} / E\right)^{3} E b / \kappa^{2}
\end{aligned}
$$

where the moment of inertia per flange is $I=b h^{3} / 12$

The above integration leads to the following moment relations:

$$
\begin{gathered}
M=M_{y} \kappa_{n} \text { if } \kappa_{n}<1 \\
M=M_{y}\left(\left(1.5-\hat{E}_{t}\right)-0.5\left(1-\hat{E}_{t}\right) / \kappa_{n}^{2}+\hat{E}_{t} \kappa_{n}\right) \text { if } \kappa_{n} \geq 1 \\
\text { where } \hat{E}_{t}=E_{t} / E, M_{y}=\sigma_{y} b h^{2} / 6, \kappa_{n}=\left(E h / 2 \sigma_{y}\right) \kappa
\end{gathered}
$$

Interpretation of (5a), (5b) leads to following conclusions:

- $\quad$ The steel section yields when $\kappa_{n}=1 \Rightarrow M=M_{y}$

- The maximum moment that can be reached is $1.5 M_{y}$ (corresponds to $E_{t}=0$ ) which leads to an elasticperfectly plastic behavior.

- In the elastic range, $M=E I \kappa$
- Deformations are obtained by integration of the moment-curvature relations.

For equilibrium to be maintained, moments from (5a) and (5b) should balance the bending moments from externally applied loads. These moments for the overhanging beam case shown in Fig. 1 are obtained from static analysis and defined as follows:

$$
\begin{array}{ll}
M=(L /(L-a)) \times(x / L) P a & 0 \leq x \leq(L-a) \\
M=(L / a-x / a)) \times P a & (L-a) \leq x \leq L
\end{array}
$$

\section{Interval of Yielding}

Yielding initiates in an interval that surrounds the interior support (region of maximum moment in Fig. 1). This region is defined as: $x_{L} \leq x \leq x_{R}\left(x_{L}\right.$ corresponds to the left side while $x_{R}$ corresponds to the right side of the intermediate support). The yield interval is quantified by setting the moments in the left $\left(x_{L}\right)$ and right $\left(x_{R}\right)$ regions equal to the yield moment $M_{y} \Rightarrow$

$$
\begin{aligned}
& x_{L}=(L-a) M_{y} / P a \\
& x_{R}=\left(1-M_{y} / P L\right) L
\end{aligned}
$$

Consequently, for the derivation of deformations, the beam can be subdivided into 4 intervals from left to right:

- An elastic region from the left support to the point where yield initiates before the middle support $(0 \leq x$ $\left.\leq x_{L}\right)$.

- An inelastic region from the point of yield to the interior support $\left(x_{L}<x \leq(L-a)\right)$.

- An inelastic region from the interior support to the point where yield initiates in the overhang after the interior support $\left((L-a)<x \leq x_{R}\right)$.

- An elastic interval from the point where yield initiates in the overhang to the tip of the cantilever $\left(x_{R}\right.$ $<x \leq L)$.

In each of these intervals, deformations can be obtained by integration of curvature $\varepsilon=\kappa y$.

\section{Derivation of Deformations}

Deformations are derived in the elastic and inelastic portions. In the elastic portions $\left(0 \leq x \leq \mathrm{x}_{\mathrm{L}} ; x_{R} \leq x \leq L\right)$, since the bending moment diagram follows a straight line shape, it can be written in the following format (the curvature is $\kappa=$ $M / E I)[4]$ :

$$
M=M_{y}\left(C_{0}+C_{l} x / L\right)=E I \kappa=\left(E b h^{3} / 12\right) \kappa
$$

The deformation is obtained by double integration of curvature:

$$
\begin{gathered}
u(x)=\iint \kappa d x= \\
\left(C_{0}\left(\frac{x}{L}\right)^{2}+\frac{1}{3} C_{l}\left(\frac{x}{L}\right)^{3}+C_{s t 1}+C_{s t 2}\left(\frac{x}{L}\right)\right) \times\left(\frac{L}{h}\right)^{2} \times\left(\frac{\sigma_{y}}{E}\right) \times h
\end{gathered}
$$


In the inelastic portions $\left(x_{L}<x<x_{R}\right)$, curvature is obtained from strain variation $(\kappa=\varepsilon / y)$. From $(5 \mathrm{~b})$, setting $\hat{E}_{t}=0$ (elastic-perfectly plastic behavior) $\Rightarrow$

$$
M=M_{y}\left(C_{0}+C_{l} x / L\right)=M_{y}\left(1.5-0.5 / \kappa_{n}^{2}\right)
$$

The deformation is obtained by double integration of curvature [4]:

$$
\begin{aligned}
& u(x)=\iint \kappa d x= \\
& \left(\frac{2}{3}\left(\frac{3-2 C_{0}-2 C_{l}\left(\frac{x}{L}\right)}{C_{l}^{2}}\right)^{3 / 2}+C_{s t l}+C_{s t 2}\left(\frac{x}{L}\right)\right)\left(\frac{L}{h}\right)^{2}\left(\frac{\sigma_{y}}{E}\right) h
\end{aligned}
$$

For the four intervals of yielding defined in the previous section, there exists four deformation functions each with two unknowns, that is a total of eight unknowns. This requires eight sets of conditions defined as follows: 1) deformation at the left support $(x=0)$ is zero, 2 and 3$)$ deformation and slope compatibility at the point of yield in the left span $\left.\left(x_{L}\right), 4\right)$ and $5)$ deformation at the intermediate support $(x=L-a)$ is zero (applies to both equations in left and right interval), 6) slope compatibility at the intermediate support $(x=L-a), 7)$ and 8) deformation and slope compatibility at the point of yield in the right span $\left(x_{R}\right)$.

\section{E. Closed Form Solutions}

The closed form solutions for non-linear deformations $(u)$ are presented for the four intervals of yield as a function of the following parameters:

- $x_{n}=x / L\left(0 \leq x_{n} \leq 1\right)$

- $\quad a_{n}=a / L\left(0 \leq a_{n} \leq 1\right)$

- $M_{y}=\sigma_{y} b h^{2} / 6$ (yielding occurs when $M=M_{y}$ )

- $m=P a / M_{y}(0 \leq m \leq 1.5$, inelastic deformation occurs for $1<m \leq 1.5)$

- $R_{n}=\left(\sigma_{\mathrm{y}} / E\right) \times(L / h)^{2}$

$0 \leq x_{n} \leq\left(1-a_{n}\right) / m$

$\frac{u}{h}=R_{n}\left(\frac{-1}{3 m^{2}}\left(-1+a_{n}\right)(10-6 \sqrt{3-2 m}+(-9+4 \sqrt{3-2 m}) m) x_{n}+\frac{m x_{n}{ }^{3}}{3-3 a_{n}}\right)$

\section{$\left(1-a_{n}\right) / m \leq x_{n} \leq\left(1-a_{n}\right)$}

$\frac{u}{h}=\frac{1}{3 m^{2}} 2 R_{n}\left(\begin{array}{l}-5\left(-1+a_{n}\right)^{2}-\left(-1+a_{n}\right)(5-3 \sqrt{3-2 m}+2 \sqrt{3-2 m m}) x_{n}+ \\ \left(-1+a_{n}\right)^{2}\left(3+\frac{2 m x_{n}}{-1+a_{n}}\right)^{1.5}\end{array}\right)$

$$
\left(1-a_{n}\right) \leq x_{n} \leq\left(1-a_{n} / m\right)
$$

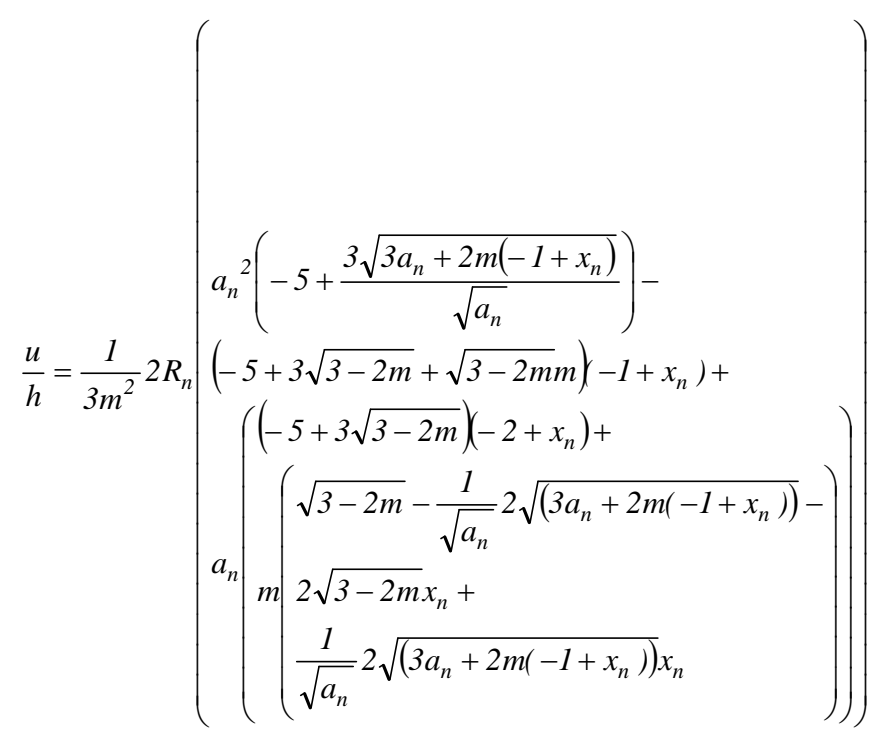

$\left(1-a_{n} / m\right) \leq x_{n} \leq 1$

$$
\frac{u}{h}=-\frac{1}{3 a_{n} m^{2}} R_{n}\left(\begin{array}{l}
2 a_{n}(-5+3 \sqrt{3-2 m}+\sqrt{3-2 m} m)\left(-1+x_{n}\right)+ \\
m^{3}\left(-1+x_{n}\right)^{3}+ \\
a_{n}{ }^{2}\left(-2(-5+3 \sqrt{3-2 m})\left(-2+x_{n}\right)+\right. \\
\left.m\left(9-2 \sqrt{3-2 m}-9 x_{n}+4 \sqrt{3-2 m} x_{n}\right)\right)
\end{array}\right)
$$

For the beam in the elastic range $(m<1)$, elastic deformations are determined as follows:

$0 \leq x \leq(L-a)$

$$
u=\frac{\operatorname{aPx}\left(-(a-L)^{2}+x^{2}\right)}{6 E I(a-L)}
$$

$(L-a)<x \leq L$

$$
u=-\frac{P(a-L+x)\left(-(L-x)^{2}+a(L+x)\right)}{6 E I}
$$

\section{Application}

The equations derived are used to determine the deformed shape of a steel beam that has a flange width $h=30 \mathrm{~cm}$ and a flange thickness $b=3 \mathrm{~cm}$ shown in Fig. 2. The length of the beam is $L=10 \mathrm{~m}$ and the overhanging portion is $a=5 \mathrm{~m}$ from Fig. 1. The load applied at the tip of the beam cantilever is $P=$ $100 \mathrm{kN}$ (that is the load for the two flanges e.g. $50 \mathrm{kN}$ per flange). The beam has a yield stress $\sigma_{y}$ of $405 \mathrm{MPa}$.

\section{A. Parameters}

Parameters are first defined as follows (per flange):

- $a_{n}=5 / 10=0.5$

- $M_{y}=\sigma_{y} b h^{2} / 6=405000 \times 0.03 \times 0.3^{2} / 6=182.3 \mathrm{kN}-\mathrm{m}$. 
- $\quad m=P a / M_{y}=50 \times 5 / 182.3=1.37<1.5$.

- $R_{n}=\left(\sigma_{\mathrm{y}} / E\right) \times(L / h)^{2}=(405 / 200,000) \times(10 / 0.3)^{2}=2.25$.

The maximum moment $M$ due to $P=100 \mathrm{kN}$ is obtained at the intermediate support as $M=P a=100 \times 5=500 \mathrm{kN}-\mathrm{m}$ for the two flanges e.g. $250 \mathrm{kN}-\mathrm{m}$ per flange $>M_{y}=182.3 \mathrm{kN}-\mathrm{m} \Rightarrow$ inelastic analysis prevails.

The interval of yield is obtained using (7a) and (7b) as follows:

$$
\begin{gathered}
x_{L}=(L-a) M_{y} / P a=(10-5) \times 182.3 /(50 \times 5)=3.65 \mathrm{~m} \\
x_{R}=\left(1-M_{y} / P L\right) L=(1-182.3 /(50 \times 10)) \times 10=6.36 \mathrm{~m} .
\end{gathered}
$$

\section{B. Deformations}

Deformations are obtained using (12) to (15) (during loading) and (16) and (17) during unloading. To avoid cluster, the deformed shapes during loading and unloading are plotted in Fig. 4 below. The residual deformed shape after removing the load (obtained by subtracting (16) and (17) from (12) to (15) is also shown.

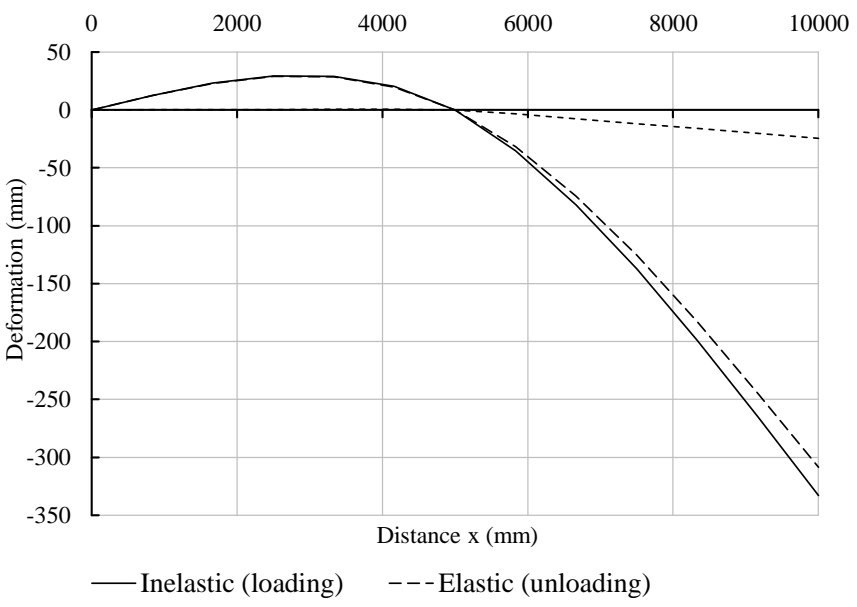

Figure 4. Deformations along the beam length (loading and unloading)

Fig. 4 shows that the maximum deformation at the tip of the cantilever at maximum load is around $330 \mathrm{~mm}$. The residual deformation is approximately $25 \mathrm{~mm}$.

Residual deformations are then used to draw the final deformed shape of the steel beam with respect to a straight line that joins its ends. This is shown in Fig. 5. It can be seen that deformations are symmetrical with respect to midspan $(x$ $=5 \mathrm{~m})$. This is attributed to the fact that the overhanging length $a$ is equal to half the span length $(L / 2=5 \mathrm{~m})$. The maximum ordinate at midspan is calculated as $12.2 \mathrm{~mm}$. A circular shape is also plotted in Fig. 5 based on the maximum ordinate at midspan.

From Fig. 5, the correlation between the circular shape and the deformed shape is adequate. Variations between the ordinates are within $60 \%$ towards the beam ends and within $90 \%$ closer to midlength. Note that this is not obvious in Fig. 5 due to inconsistency in the scale between the vertical and horizontal axis (the maximum variation between the ordinates is quantified as $2.7 \mathrm{~mm}$ at $x=L / 4$ and $3 L / 4$ ).

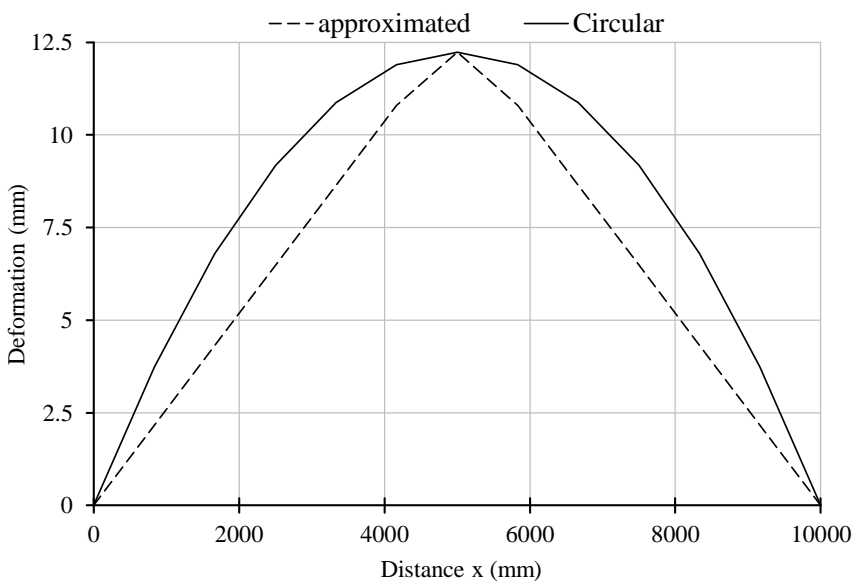

Figure 5. Deformed shape based on ordinates compared to a smooth curve

In summary, the deformed shape due to point bending at the tip of the cantilever of the overhanging beam can be used to idealize a smooth curve.

\section{v. Conclusions}

This paper develops a generalized analytical solution for point bending overhanging steel beams about its weak axis. Closed form solutions for deformations in the inelastic range and elastic range are derived for this purpose. The equations derived are then used to draw the deformed shape of steel beams for the simple case where the overhanging length is equal to half the span length of the beam.

An illustrative example is included. It is shown that the residual deformed shaped after removing the bending load is closer to a smooth circular curve. Consequently, the procedure developed in this paper can be used as a tool for fabricating circular curves in steel beams.

\section{Acknowledgment}

The author would like to express his gratitude to professor Nabil Fares at the University of Balamand for helping out in deriving the closed form solutions using Mathematica.

\section{References}

[1] Bjorhovde, R., "Cold bending of wide-flange shapes for construction", AISC Engineering Journal 2006, vol.43, no.4, pp.271-286.

[2] Gergess, A. and Sen, R., "Non-Linear Analysis of Wide Flange Steel Beams Subjected to Point Bending", in B.H.V. Topping, P. Iványi, (Editors), "Proceedings of the Twelfth International Conference on Computational Structures Technology", Civil-Comp Press, Stirlingshire, UK, Paper 16, 2014. doi:10.4203/ccp.106.16.

[3] Gergess, A. and Sen, R. Curving structural steel girders by two-point bending. Journal of Constructional Steel Research, 2016. 122, 511-519.

[4] Popov E.V., "Mechanics of Materials", Prentice-Hall Inc., Englewood Cliffs, New Jersey, $2^{\text {nd }}$ Ed., 1976 (pages 135-142, 377-393).

[5] Barnshaw, R., "Bending considerations in steel construction". Modern Steel Construction (MSC), 2009, October, pp. 42- 44.

[6] ANSI/AISC 360-10, Specification for Structural Steel Buildings, $4^{\text {th }}$ printing. 2015, American Institute of Steel Construction, Chicago, Illinois, USA. 
Proc. of the Fifth Intl. Conf. Advances in Civil, Structural and Mechanical Engineering - CSM 2017 Copyright (C) Institute of Research Engineers and Doctors, USA .All rights reserved.

ISBN: 978-1-63248-132-0 doi: 10.15224/ 978-1-63248-132-0-40

About Author (s):

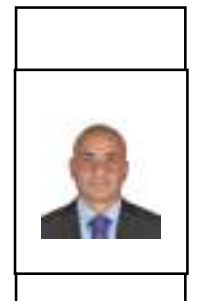

The deformed shape due to point bending at the tip of the cantilever of the overhanging beam can be used to idealize a smooth curve. 